\title{
Investigation of Constant Volume and Constant Flux Initial Conditions on Bidensity Particle-Laden Slurries on an Incline
}

\author{
Dominic Diaz*, Jessica Bojorquez, Joshua Crasto, Margaret Koulikova, Tameez Latib, Aviva Prins, Andrew Shapiro, Clover Ye, \\ David Arnold, Clandia Falcon, Michael R. Lindstrom, Andrea L. Bertozzi \\ Department of Mathematics, University of California, Los Angeles, CA \\ https://doi.org/10.33697/ajur.2019.029 \\ Students:dominicdiaz@g.ucla.edu*, jessicarbojorquez@gmail.com, jcrasto@ucla.edu,m.koulikova@ucla.edu, \\ tameezlatib@gmail.com,aviva@avivaprins.com,alshap1010@yahoo.com,clover1869@ucla.edu \\ Mentors:darnold@math.ucla.edu, cfalcon@math.ucla.edu,mikel@math.ucla.edu,bertozzi@math.ucla.edu
}

\begin{abstract}
Particle-laden slurries are pervasive in both natural and industrial settings, whenever particles are suspended or transported in a fluid. Previous literature has investigated the case of a single species of negatively buoyant particles suspended in a viscous fluid. On an incline, three distinct regimes emerge depending on the particle concentration and inclination angle: settled (where particles settle and there is a pure fluid front), well-mixed (where particle concentration is constant throughout), and ridged (where a particle-rich ridge leads the flow). Recently, the same three regimes were also found for constant volume two species bidensity slurries. We extend the literature on bidensity slurries by presenting results on constant volume and a new type of initial condition: constant flux, where slurry is pumped onto the incline at a constant rate. We present front positions of the slurries and compare them to theoretical predictions. In addition, height profiles (film thicknesses) are also presented for the constant flux case, showing the distinct behavior of the ridged regime. We find that for constant flux conditions the settled regime forms for small particle volume fractions and inclination angles while the ridged regime forms for large corresponding values. Intermediate values of these two parameters are shown to produce a well-mixed regime.
\end{abstract}

\section{KEYWORDS}

Thin Films; Particle-Laden Flow; Multiphase Fluids; Interfacial Flows; Particle Segregation

\section{INTRODUCTION}

A thin film of viscous fluid flowing down an incline has been observed experimentally and analyzed mathematically. ${ }^{1}$ In nature, instead of being observed as pure fluids, fluids are more often observed as mixtures of various fluids and other particles. Expanding the work done by Huppert, ${ }^{1}$ many studies have investigated thin, particle-laden slurries, which are mixtures of particles suspended in a fluid. Thin films of monodisperse particle-laden slurries (viscous fluids mixed with only a single type of particle) have been extensively studied both theoretically and experimentally. ${ }^{2-8}$ The mathematical equations governing the flow of these slurries become increasingly complex as we add more species of particles to the mixture, but the solutions become more relevant to the fluids we observe in industrial and natural settings (such as in spiral particle separators, landslides, and mud). ${ }^{9-13}$

Bidensity particle-laden slurries, which are the focus of this study, are mixtures of two species of particles-with different densities and similar diameters-suspended in a viscous fluid. When an initially uniformly mixed slurry flows down an incline, the particles experience a fast initial equilibration period (this has been investigated for the monodisperse case, ${ }^{14}$ but we assume similar results apply to the bidensity case we investigate here). During this period, particles in the mixture experience two significant and opposing effects whose relative strengths determine the long term behavior of the particles in the direction normal to the incline: sedimentation due to gravity (towards the incline surface) ${ }^{15,16}$ or shear-induced migration, ${ }^{17}, 18$ which leads particles away from areas of high shear rate and high particle concentra- 
tion (away from the incline surface). Depending on which effect dominates, there are three possible regimes that can arise: ridged, well-mixed, and settled. The ridged regime occurs when shear-induced migration dominates gravitational settling, and is identified by a high concentration of particles on the front edge of the fluid, forming a leading ridge. In the settled regime, where gravity dominates shear-induced migration, particles form a sediment at the bottom of the fluid as it flows down the track. Within this regime for a bidensity slurry, separation between different particles and fluid can be observed. The well-mixed regime, a transient state between the other two regimes, ${ }^{4}$ is formed when neither of these effects dominate and particles stay evenly distributed throughout the fluid.

These three regimes have been extensively studied in the monodisperse (single particle species) case for a constant volume of fluid ${ }^{3-5,8}$ and in the bidensity case for a constant volume of fluid. ${ }^{19}$ Murisic et al. ${ }^{4}$ constructed a regime phase diagram for a specific fluid viscosity and particle diameter with varying particle volume fraction and angle of inclination. They find that the ridged regime tends to form for high inclination angle and particle concentration while the settled regime tends to form for small inclination angle and particle concentration. This result comes from the effect of shear-induced migration increasing with greater angle and/or particle concentration. The bidensity, constant volume case was then studied theoretically ${ }^{20,21}$ and experimentally ${ }^{19}$. In particular, Lee et al. observes the formation of the three regimes for various inclination angles and heavy-to-light particle ratios. ${ }^{19}$

In addition to observing regime formation of thin viscous slurries on an inclined surface, various studies have also investigated how the fluid's average front position evolves with time. For a constant volume of viscous fluid, Huppert ${ }^{1}$ finds that the fluid's average front position evolves with a behaviour asymptotic to $t^{1 / 3}$ (where $t$ is time). In both the monodisperse ${ }^{5}$ and bidensity ${ }^{21}$ case for a finite volume of fluid, it has been shown that the average front position is still asymptotic to $t^{1 / 3}$.

In the current study, we first extend the constant volume results presented in Lee et al. ${ }^{19}$ and Wong et al. ${ }^{21}$ by explicitly presenting experimentally found front velocities for bidensity particle-laden slurries. We then directly extend these results to a new type of initial condition: a constant flux condition, where slurry is pumped onto the top of the inclined surface at a constant rate. For this initial condition, we consider the results of Lee's model for particle distribution normal to the place of the $\operatorname{track}^{20}$ and expect similar regime phenomena to arise. We present the experimentally found front positions and regime classifications for bidensity, constant flux slurries to examine the effect the initial condition has on the dynamics of the slurry. While much of the literature focuses on front position and regime classification, we additionally present height profiles of the bidensity, constant flux slurries.

\section{METHODS AND PROCEDURES}

\section{Materials}

The mixtures used in our experiments consisted of glass and ceramic beads suspended in a silicone oil. The particles, manufactured by Ceroglass, are described in Table 1. There is a slight disparity in the average diameter of the two particles although we assume that their diameters are the same for the purposes of this paper. For constant volume experiments, red ceramic beads were used to emphasize the separation of distinct fronts, while for constant flux experiments, red ceramic beads were used in some experiments and white ceramic beads were used in others. The viscous fluid we used was polydimethylsiloxane (see the last row of Table 1 for properties).

\begin{tabular}{|c|c|c|c|}
\hline Material & Density & Color & Other Properties \\
\hline GSB-7 glass beads & $2.5 \mathrm{~g} / \mathrm{cm}^{3}$ & white & diameter: $0.18-0.25 \mathrm{~mm}$ \\
\hline SLZ-2 ceramic beads & $3.8 \mathrm{~g} / \mathrm{cm}^{3}$ & red & diameter: $0.125-0.25 \mathrm{~mm}$ \\
\hline polydimethylsiloxane (PDMS) & $0.971 \mathrm{~g} / \mathrm{cm}^{3}$ & clear & kinematic viscosity: $10 \mathrm{~cm}^{2} / \mathrm{s}$ \\
\hline
\end{tabular}

Table 1. Materials used in our experiments. The density and diameter are the same for all experiments. Note that for some of the constant flux experiments, the color of the ceramic particles was white. 


\section{Conducting the experiments}

In this study we performed a series of experiments on gravity driven bidensity slurries flowing on an incline. We varied four parameters for each experiment: inclination angle of the track $\alpha$, the volume fraction ratio of lighter density particles (glass) to heavier density particles (ceramic) $\chi$, total particle volume fraction $\phi$, and the initial condition (either constant volume or constant flux). We define the volume fraction of glass and ceramic particles respectively as:

$$
\phi_{g}=\frac{V_{g}}{V_{g}+V_{c}+V_{f}}, \quad \phi_{c}=\frac{V_{c}}{V_{g}+V_{c}+V_{f}} .
$$

Equation 1.

Where $V_{g}$ is the volume of glass particles, $V_{c}$ is the volume of the ceramic particles, and $V_{f}$ is the volume of fluid in the mixture. We additionally define the total particle volume fraction as the sum of the two particle volume fractions:

$$
\phi=\phi_{g}+\phi_{c} .
$$

Equation 2.

We define the volume fraction ratio $\chi$ as the ratio of $\phi_{g}$ to the total volume fraction, $\phi_{g}+\phi_{c}$ :

$$
\chi=\frac{\phi_{g}}{\phi_{g}+\phi_{c}}=\frac{V_{g}}{V_{g}+V_{c}} .
$$

Equation 3.

The two initial conditions-constant volume or constant flux-are defined in terms of their nondimensional height by the following two equations, respectively:

$$
h(x, t=0)=\left\{\begin{array}{ll}
0 & \text { if } x<-l \\
1 & \text { if }-l \leq x \leq 0, \\
0 & \text { if } x>0
\end{array} \quad \text { and } \quad h(x, t=0)= \begin{cases}1 & \text { if } x \leq 0 \\
0 & \text { if } x>0\end{cases}\right.
$$

where $x$ and $t$ are nondimensional position and time, respectively. We define $l$ as some nondimensional length, $x=$ 0 as the location where the slurry starts to flow, and $t=0$ as the time in which the slurry starts to flow. Schematic diagrams of these two initial conditions are presented in Figure 1.
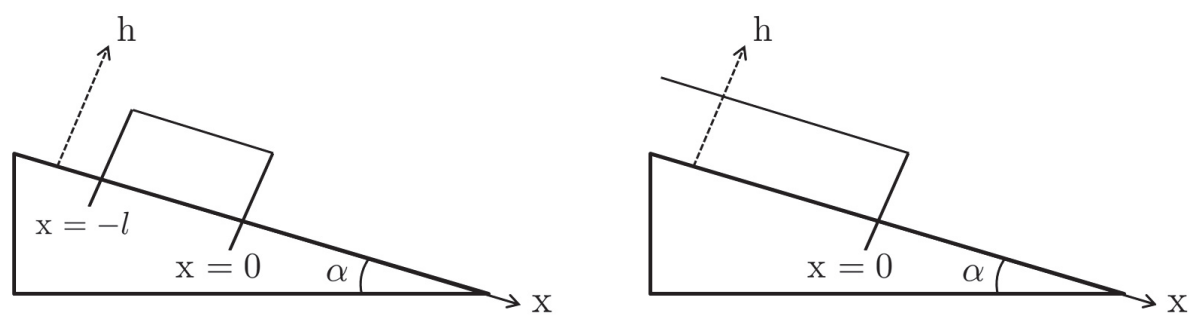

Figure 1. Schematic diagram of the constant volume (left) and constant flux (right) initial conditions.

To perform our experiments, we used an acrylic track (length $90 \mathrm{~cm}$ and width $14 \mathrm{~cm}$ ) with an adjustable angle, $\alpha$, (see Figure 2) that was adjusted to either $20^{\circ}$ or $50^{\circ}$ for each experiment. In the constant volume experiments, the region on the track behind the gate (see Figure 2, left) was filled with $80 \mathrm{~mL}$ of well-mixed slurry. The gate was then quickly lifted to allow the slurry to flow down the track. In the constant flux experiments, a weir was placed near the top of our track (see Figure 2, right). On the portion of the track above the weir, we constantly pumped well-mixed slurry onto the track. The purpose of the weir was to ensure that the slurry flowed onto the track evenly along the width of the track (y-axis). The volume of slurry entering the track over time was constant at around $3 \mathrm{~cm}^{3} / \mathrm{s}$ for $\phi=0.25$ experiments and around $0.75 \mathrm{~cm}^{3} / \mathrm{s}$ for $\phi=0.45$ experiments. Because of the weir the volumetric flux, or flow rate per unit surface, was constant at the top of the track. The length of the track (in the x-direction) is much longer than the width of the track (in the y-direction) but for the purposes of our analysis we ignore edge effects due to the walls of the track. 


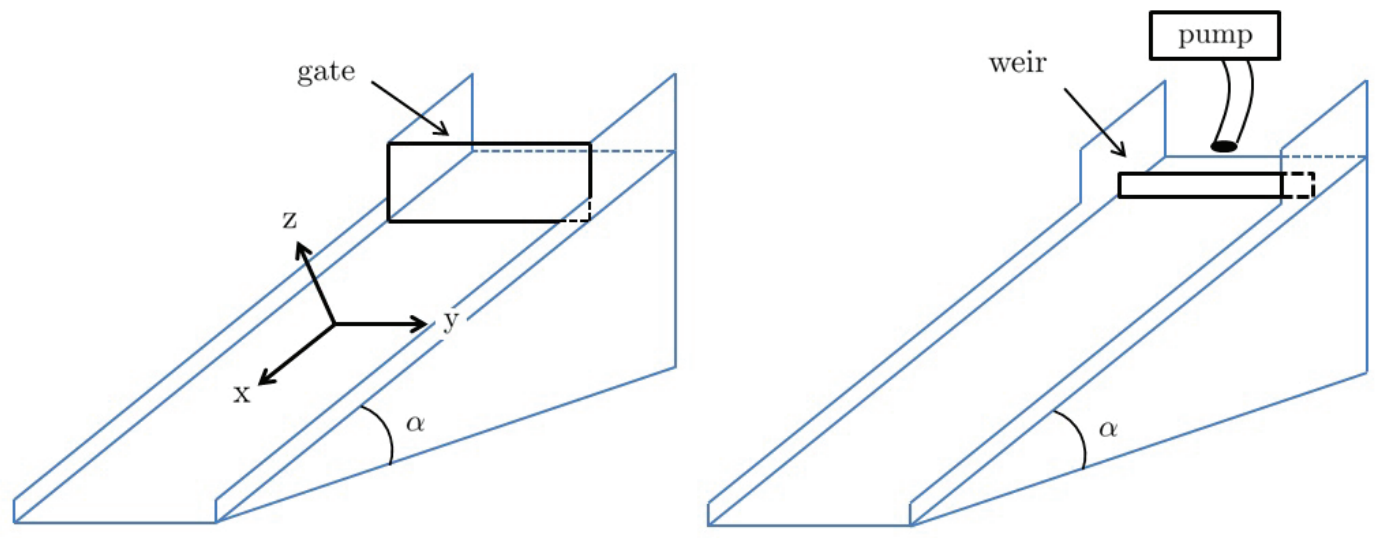

Figure 2. Schematic of our experimental setup. The left image depicts the setup for the constant volume experiments. The right image depicts the setup for the constant flux experiments. The coordinate system is kept constant for both initial conditions.

\section{Data Analysis}

We quantified the positions of the fronts and the slurry's film thickness (height profile) along the $\mathrm{x}$-axis. Both methods utilized code written in MATLAB, which is summarized in Supplement A. In order to find the position of the individual fronts, a camera was set up above the track with the face of the lens parallel to the plane of the track, 0.5 to $1 \mathrm{~m}$ away. The front position was computed for individual frames of the resulting video after cropping out the background and the edges of the width of the track. We cropped out these edges to minimize the contamination of edge drag (which slows down the slurry close to the side walls [see Figure 3]) on our front position data since theoretical predictions do not account for wall effects. Fronts are identified using RGB values for each vertical strip of the frame. Figure 4 shows individual frames of a settled and well-mixed experiment.

We also investigated the film thickness of the flow along the x-axis (we present pseudocode in Algorithm 2 of Supplement A). To do this, we first set up our camera next to the track such that the camera lens was pointed towards the track. We then shined a laser line on the middle of the track so that the line was parallel to the length of the track. The camera lens face was then made to be perpendicular to the incline so that the camera could pick up the laser line shining on the incline and its deflection due to the presence of some calibration object or the slurry on the incline. Using calibration images of the empty track and of the track with an object of known height resting on it, we were able to process the video of the experiment to obtain the film thickness. As the slurry flowed down the track, the laser line's position in each frame was deflected by the presence of the slurry. This deflection was computed using MATLAB, in $\mathrm{mm}$, which resulted in figures such as Figure 9.

\section{RESULTS AND DISCUSSION}

Our results are divided into three sections, based on the initial condition and the type of data presented: front tracking and regime classification for constant volume experiments, front tracking and regime classification for constant flux experiments, and film thickness for constant flux experiments. For each experiment, the inclination angle $\alpha$, volume ratio $\chi$, and total particle volume fraction $\phi$ were varied. Based on previous research, we expect the ridged regime to form for large $\alpha$ and $\phi$ and the settled regime to form for small $\alpha$ and $\phi$.

\section{Constant volume: front tracking and regime classification}

We conducted experiments with a constant volume of slurry moving down an incline positioned at inclination angle, $\alpha$, with particle volume ratio, $\chi$. Pictures of the experiments are shown in Figure 3. With the exception of the $\chi=$ 0.5 experiments (which had $\phi$ values of 0.25 and 0.4 ), all constant volume experiments had a total volume fraction of $\phi=0.45$; this allows us to present front speeds for slurries with three different $\phi$ values. We chose to use distinct $\phi$ values for the intermediate particle volume ratio as opposed to the more extreme volume ratios that are tested because 
we wanted to compare the observed regimes for experiments with only the inclination angle or the volume fraction ratio being changed significantly. In terms of the regime that formed, the two angles produced different regimes for each $\chi$ value (see Table 2 for the classifications of the constant volume experiments). If we look at the two $\chi=0.389$ experiments, the smaller angle produced a settled slurry while the larger angle produced a ridged slurry. Angle was not the only parameter that affected the regime. If we compare the top left experiment with the top right experiment, we see the volume fraction ratio influenced the regime of the slurry. If we compare all three of the bottom experiments, we expect that the middle experiment would have also been ridged for a $\phi$ value of 0.45 but because the slightly lower $\phi$ value of 0.4 produced a well-mixed slurry, we can also conclude that the total particle volume fraction affects the regime. It appears that all three parameters $-\alpha, \chi$, and $\phi-$ affect the regime that is formed and this result agrees with the findings of Lee et al. ${ }^{19}$

Figure 4 provides the evolution of two sample flows. In Figure 4a (a settled slurry), three distinct fronts formed for the PDMS, glass beads, and ceramic beads. In Figure $4 \mathrm{~b}$ (a well-mixed slurry), the fluid and the particle fronts remained together. Using the front tracking technique described in the Methods and Procedures section we produce the blue and green dots in Figure 4 which correspond to individual particle front positions. The leftmost two images of Figure 4a do not track the particle fronts well (this occurs in the beginning of most of our settled experiments due to variation of particle front color as the particles begin to settle). This error does not affect our analysis since theory only predicts asymptotic behavior after the slurry has reached equilibrium (i.e. towards the end of the experiment) and thus front tracking early in the experiment was not as important as later on in the experiment. Notice that in Figure 4a, the fluid front moved faster than the particle fronts. With the angle and particle concentrations being so low, the particles settled to the bottom of the slurry (towards the incline surface) while the fluid flowed over the settled particles, moving faster. In Figure 4b, we do not observe this separation; the particles stayed well-mixed throughout the fluid.

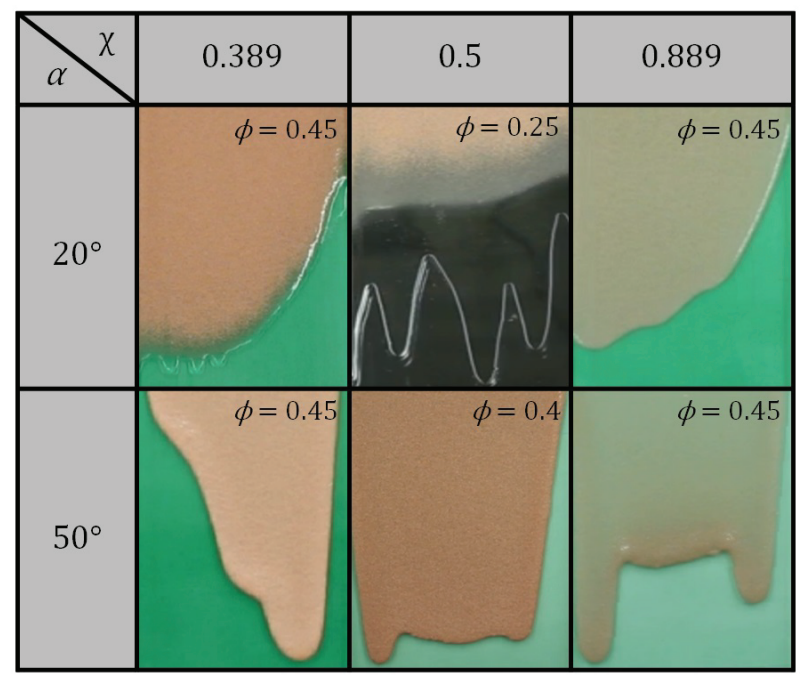

Figure 3. Experiments conducted with a constant volume of slurry. We performed six experiments in total, with three different $\chi$ values $(0.389,0.5$, and 0.889 ) and with two different angles $\left(20^{\circ}\right.$ and $\left.50^{\circ}\right)$. The total volume fraction, $\phi$, for each experiment is also presented in the top right corner of each image. Note that the $(\chi, \alpha)=\left(0.5,20^{\circ}\right)$ experiment was conducted with a black background because it showed better contrast between each of the three fronts than a green background. 


\begin{tabular}{|c|c|c|c|}
\hline$\alpha$ & 0.389 & 0.5 & 0.889 \\
\hline $20^{\circ}$ & $\begin{array}{c}\text { settled } \\
(\phi=0.45)\end{array}$ & $\begin{array}{c}\text { settled } \\
(\phi=0.25)\end{array}$ & $\begin{array}{c}\text { well-mixed } \\
(\phi=0.45)\end{array}$ \\
\hline $50^{\circ}$ & $\begin{array}{c}\text { ridged } \\
(\phi=0.45)\end{array}$ & $\begin{array}{c}\text { well-mixed } \\
(\phi=0.4)\end{array}$ & $\begin{array}{c}\text { ridged } \\
(\phi=0.45)\end{array}$ \\
\hline
\end{tabular}

Table 2. The six constant flux experiments presented in Figure 3 along with the observed regime and the total volume fraction of the fluid, $\phi$.

According to theory, we expect the front position of the fluid to have behavior asymptotic to $t^{1 / 3}$, where $t$ is time. This asymptotic behavior arises after the slurry has reached equilibrium so we fit the front position of the fluid to $x(t)=c t^{\beta}+k$ for constants $c, k$, and $\beta$ when the slurry is approximately halfway down the incline. In Table 3 , we present our fitted $\beta$ values for each of our experiments presented in Figure 3. Notice that the settled experiments have fitted $\beta$ values that are furthest away from our theoretically predicted value of $1 / 3$. In these experiments, the fluid front separates from the particle front(s) and blends in with the incline making it difficult to track (see Figure 4a). There is strong agreement between theoretical predictions and our non-settled experimental results and we suspect that we would find better agreement for the settled experiments with either a different fluid color or a different front tracking method for settled experiments. One factor that may have had an impact on our fitted values is the presence of edge drag. Although we cropped our experimental videos to minimize its impact on our analysis, edge drag was present to varying degrees in every constant volume experiment depicted in Figure 3 and could also have been a source of the slight deviations of our results from theoretical predictions. In Figure 5a, we present our experimental data against the best fit line for the $(\chi, \alpha)=\left(0.389,50^{\circ}\right)$ experiment. For most constant volume experiments, the data fits well with the fitted equations as seen in this figure. When we fit for $\beta$ values with larger error, the experimental data does not align with the fitted equation as well as it does in Figure 5a. We generally find that the fitted experimental front positions agree, within error, with the theoretically predicted behavior.
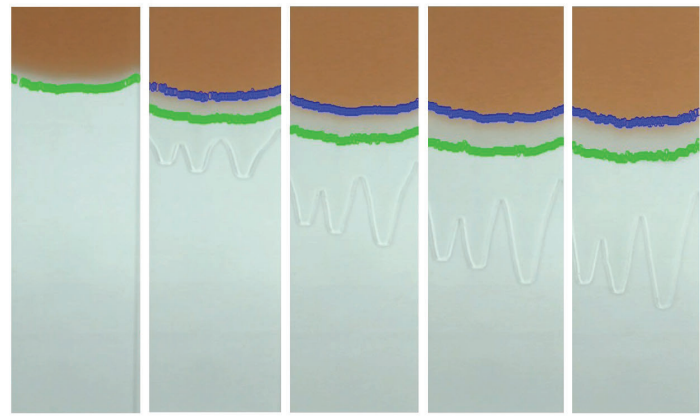

(a) $(\chi, \alpha)=\left(0.5,20^{\circ}\right)$ at 70 second intervals
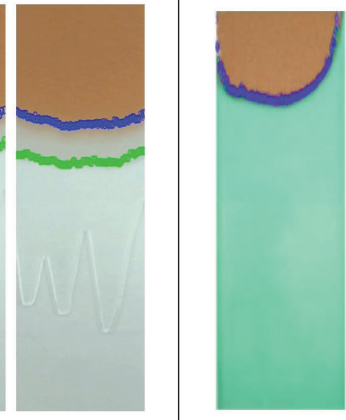
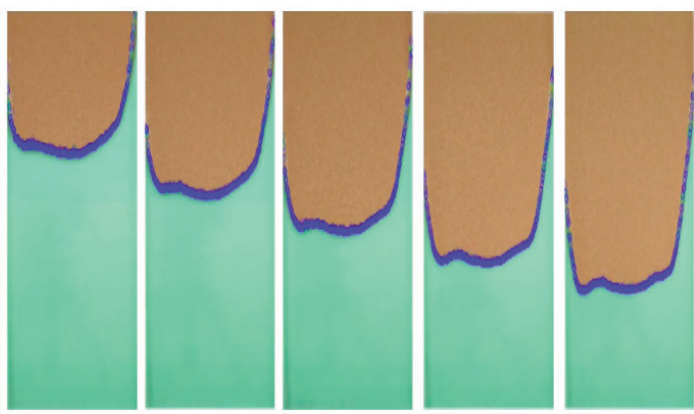

(b) $(\chi, \alpha)=\left(0.5,50^{\circ}\right)$ at 12 second intervals

Figure 4. Processed video screenshots of the slurry flowing down the track. The light green and blue dots track the front position of the glass beads and ceramic beads, respectively. Throughout the course of our study, we experimented with various colored backgrounds that provided the best contrast between the fronts and the background color. We settled with a green background but present one of our early background color trials (a white background) in (a) for two reasons: (1) to show how the clear fluid blends in with the background making the fluid front difficult to track and (2) because this specific experiment produced the most symmetric and clear example of a settled slurry. Note that the first image in (a) only includes green dots since distinct fronts had not yet emerged in the experiment.

\begin{tabular}{|c|c|c|c|}
\hline$\alpha$ & 0.389 & 0.5 & 0.889 \\
\hline $20^{\circ}$ & $0.19 \pm 0.5$ & $0.22 \pm 0.05$ & $0.32 \pm 0.05$ \\
\hline $50^{\circ}$ & $0.31 \pm 0.02$ & $0.39 \pm 0.5$ & $0.38 \pm 0.07$ \\
\hline
\end{tabular}

Table 3. Fitted $\beta$ values for each of the experiments presented in Figure 3 with their respective errors ( $95 \%$ confidence intervals). Theoretically, we expect our values to be around $1 / 3$. 


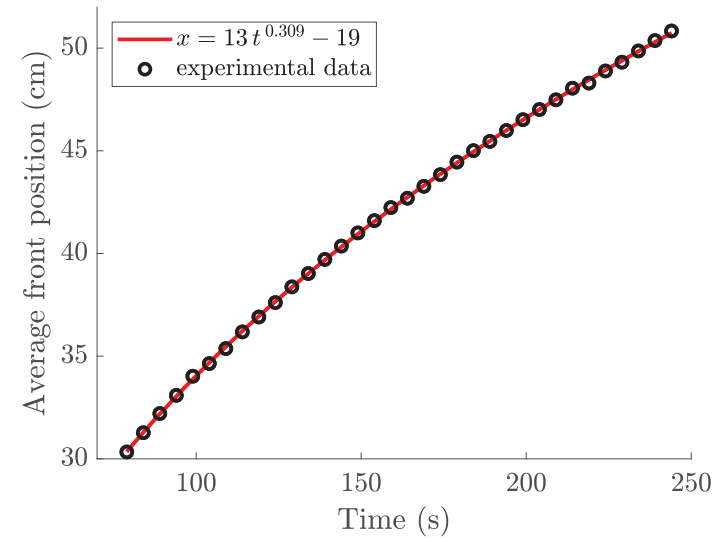

(a) constant volume fit

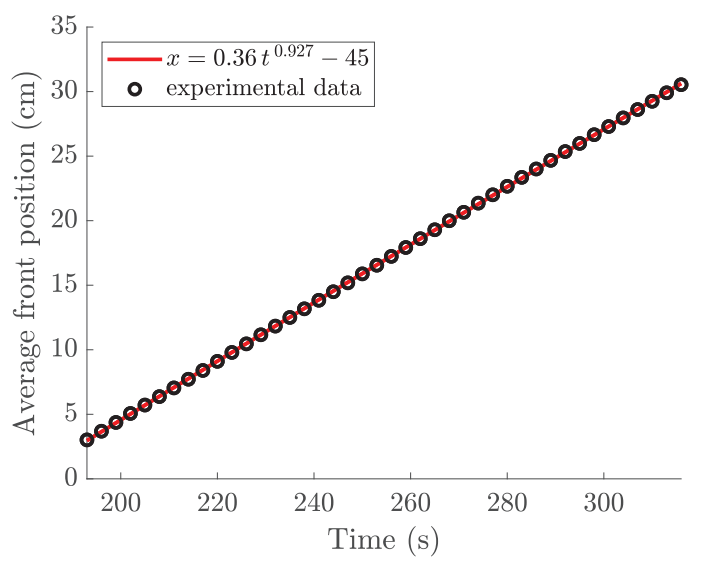

(b) constant flux fit

Figure 5. Fitted equations plotted against experimental fluid front data for a constant volume and constant flux experiment, both of which are experiments in the ridged regime. (a) Corresponds to the $(\chi, \alpha)=\left(0.389,50^{\circ}\right)$ constant volume experiment while (b) corresponds to the $(\chi, \alpha)=\left(0.378,50^{\circ}\right)$ constant flux experiment.

\section{Constant flux: front tracking and regime classification}

We conducted eight constant flux experiments at different $\chi, \alpha$, and $\phi$ values, as shown in Figure 6. In each experiment we investigated the emergence of regimes, the front position, and film thickness (in the next section).

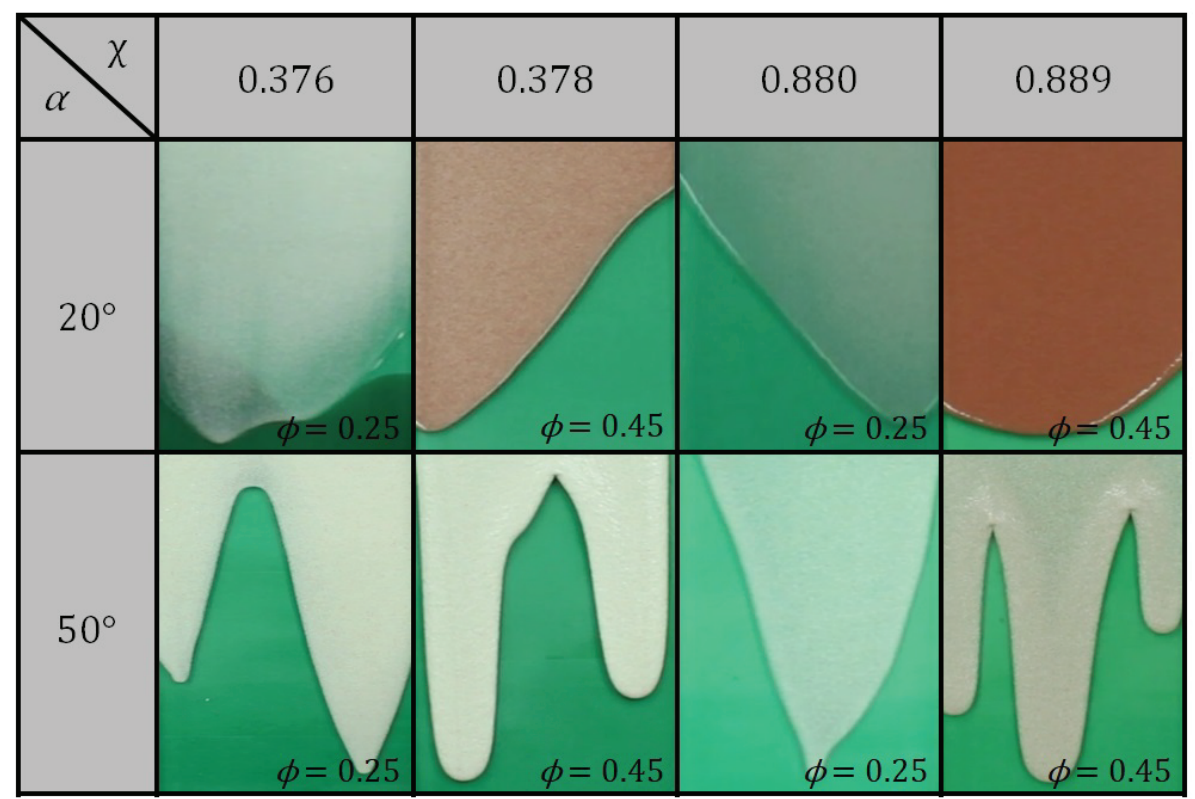

Figure 6. We performed eight constant flux experiments at four different $\chi$ values $(0.376,0.378,0.880$, and 0.889$)$ and at two different inclination angles $\left(20^{\circ}\right.$ and $\left.50^{\circ}\right)$. The $\phi$ value for each experiment is also presented in the bottom right corner. Each cell of the table is also classified by its regime in Table 4. Note that in only two of the experiments $\left((\chi, \alpha)=\left(0.378,20^{\circ}\right)\right.$ and $\left.(\chi, \alpha)=\left(0.889,20^{\circ}\right)\right)$ the ceramic particles are red and the glass particles are white while in the rest of the experiments both particle species are white.

Unlike constant volume experiments, we focus on two values of overall particle ratio $\phi: 0.25$ and 0.45 . For $\phi=0.25$, the slurries are settled at $\alpha=20^{\circ}$, but at $\alpha=50^{\circ}$, the slurry is roughly well-mixed, possibly on the verge of a ridged regime. For $\phi=0.45$ and $\alpha=20^{\circ}$, the slurries are well-mixed, possibly on the verge of a settled regime, while slurries at $\phi=0.45$ and $\alpha=50^{\circ}$ are evidently ridged. While the volume fraction ratio $\chi$ is shown to have no effect on the regime (compare each of the four sets of experiments that only differ in their $\chi$ value), the angle of inclination $\alpha$ and 
the total volume fraction $\phi$ do have an effect on the regime produced. To see the regime classification of each of the experiments in Figure 6, see Table 4. The transient well-mixed regime occurred frequently (half of our constant flux slurries were classified as well-mixed), indicating that some of the experiments may have ended prematurely. A longer track would allow our slurry to flow further and might lead to different regime classification in these experiments. We find that, similar to the constant volume experiments, large $\phi$ and $\alpha$ values produce a ridged regime while small corresponding values produce a settled regime for constant flux slurries. We also find that varying volume fraction ratio, $\chi$, alone does not have a significant impact on regime formation of constant flux experiments; this contrasts with our findings that this parameter does have a significant impact on the regime formation of constant volume experiments.

\begin{tabular}{|c|c|c|c|c|}
\hline$\alpha$ & 0.376 & 0.378 & 0.880 & 0.889 \\
\hline $20^{\circ}$ & $\begin{array}{c}\text { settled } \\
(\phi=0.25)\end{array}$ & $\begin{array}{c}\text { well-mixed } \\
(\phi=0.45)\end{array}$ & $\begin{array}{c}\text { settled } \\
(\phi=0.25)\end{array}$ & $\begin{array}{c}\text { well-mixed } \\
(\phi=0.45)\end{array}$ \\
\hline $50^{\circ}$ & $\begin{array}{c}\text { well-mixed } \\
(\phi=0.25)\end{array}$ & $\begin{array}{c}\text { ridged } \\
(\phi=0.45)\end{array}$ & $\begin{array}{c}\text { well-mixed } \\
(\phi=0.25)\end{array}$ & $\begin{array}{c}\text { ridged } \\
(\phi=0.45)\end{array}$ \\
\hline
\end{tabular}

Table 4. The eight constant flux experiments presented in Figure 6 along with the observed regime and the total volume fraction of the fluid, $\phi$.

In addition to the regime formation we investigated the fluid front position of constant flux bidensity slurries. For these experiments we again attempted to fit the front position of our fluid to an equation of the form $x(t)=c t^{\beta}+k$ for some constants $c, k$, and $\beta$ where we now expect our $\beta$ value to be 1 . We present our fitted $\beta$ values in Table $\mathbf{5}$, where we see that the $\beta$ values centered around 1 . We obtain loose agreement with theoretical predictions, with the settled experiment $(\chi, \alpha)=\left(0.376,20^{\circ}\right)$ producing the result furthest from our predictions. Again, this is likely due to the fluid front being difficult to track once it separates from the particle front(s). We suspect that using a non-clear fluid or a different fluid front tracking method would produce stronger agreement in this settled experiment. Similar to the constant flux experiments, edge drag may also have been a source of the slight deviations of our experimental results from theoretical predictions. To see an example fitted equation plotted against experimental data for a constant flux experiment, see Figure 5b. Notice that because we expect our front position to be linear (and we see linear behavior in the experimental data presented in Figure $\mathbf{5 b}$ ), if we fit the front position data to some equation $x(t)=c t+k$, then $c$ is the front speed of the slurry. For the $(\chi, \alpha)=\left(0.378,50^{\circ}\right)$ constant volume experiment (presented in Figure $5 \mathbf{b}$ ), fitting to the linear equation produces a front speed of $0.22 \pm 0.8 \mathrm{~cm} / \mathrm{s}$. As future work, we plan to use shock theory and numerical simulations to simulate the front velocity of the slurry and validate those numerical results against the experimentally found front velocities of the constant flux experiments presented in this paper.

\begin{tabular}{|c|c|c|c|c|}
\hline$\alpha$ & 0.376 & 0.378 & 0.880 & 0.889 \\
\hline $20^{\circ}$ & $0.76 \pm 0.3$ & $0.90 \pm 0.1$ & $0.96 \pm 0.1$ & $0.91 \pm 0.03$ \\
\hline $50^{\circ}$ & $1.1 \pm 0.1$ & $0.93 \pm 0.09$ & $1.18 \pm 0.08$ & $1.08 \pm 0.09$ \\
\hline
\end{tabular}

Table 5. Fitted $\beta$ values for each of the experiments presented in Figure 6 with their respective errors (95\% confidence intervals). We expected our values to be around 1 .

One interesting phenomenon that we saw in our experiments is presented in Figure 7. We noticed a folding-over phenomenon in constant flux experiments for large inclination angles and total volume fractions but never in other experiments. It was also only observed between fingers in these experiments (fingers correspond to the individual streaks of fluid that run down the track) and became most prominent towards the bottom of the track. For example, in the $(\chi, \alpha)=\left(0.889,50^{\circ}\right)$ experiment, we observed the phenomenon starting to form in the corresponding image of Figure 6 which, after some time, developed more prominently into what we present in the right image of Figure 7. 

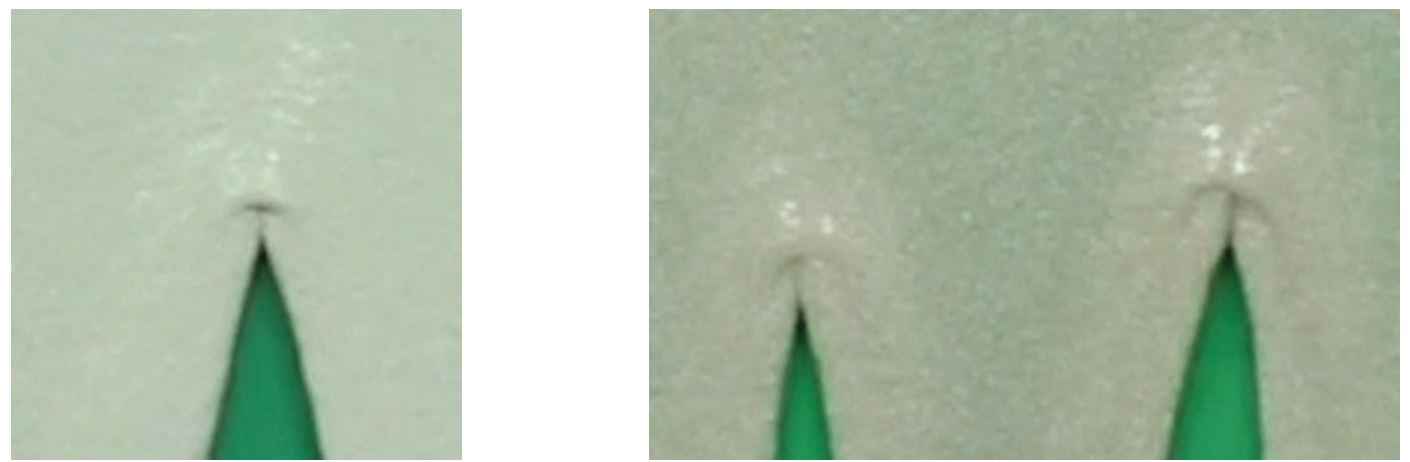

Figure 7. Two images of folding-over phenomenon occurring in constant flux experiments with large $\alpha$ and $\phi$. Both images are zoomed in images from constant flux experiments with the left one corresponding to $(\chi, \alpha)=\left(0.378,50^{\circ}\right)$ and the right one corresponding to $(\chi, \alpha)=\left(0.889,50^{\circ}\right)$.

\section{Constant flux: flm thickness}

To track the film thickness of the experiments presented in Figure 6, we implemented the film thickness tracking technique explained in the Methods and Procedures section. According to theory, we expect a distinct film thickness profile for each regime. We present the thickness of our fluid at four different times to show the evolution of the thickness for each of the three regimes in Figures 8, 9, and 10. We estimate the error in the measured film thickness to be about 0.5 $\mathrm{mm}$ (the film thickness should be smooth because of surface tension but error is introduced because of the resolution of our images). The sequences of images in Figures 8, 9, and 10 are characteristic of the thickness results for the settled, well-mixed, and ridged regimes, respectively.

In most cases, we found that the settled and well-mixed film thicknesses were similar: the smallest thickness was found at the front of the fluid and it increased after the front until a certain point where it would approximately plateau. This behavior is most clearly seen in the rightmost graph of Figures $\mathbf{8}$ and $\mathbf{9}$ although this behavior can also be seen in the other three graphs in the same figures. Considering the resolution of our images and surface tension, it is not surprising that we observe similar height profiles for the settled and well-mixed regimes. For the settled regime we expect to observe three distinct plateaus which correspond to the three fronts ${ }^{21}$ while for the well-mixed regime we expect to observe a constant height wherever slurry is present due to the constant flux initial condition. The lack of three distinct plateaus in the settled regime is likely due to the resolution of our images while the lack of a single plateau in the well-mixed regime is likely due to surface tension. The ridged regime exhibits significantly different behavior than the other two: a tall leading ridge forms and is followed by an approximately constant plateau. This behavior agrees with the behavior of constant volume ridged slurries.
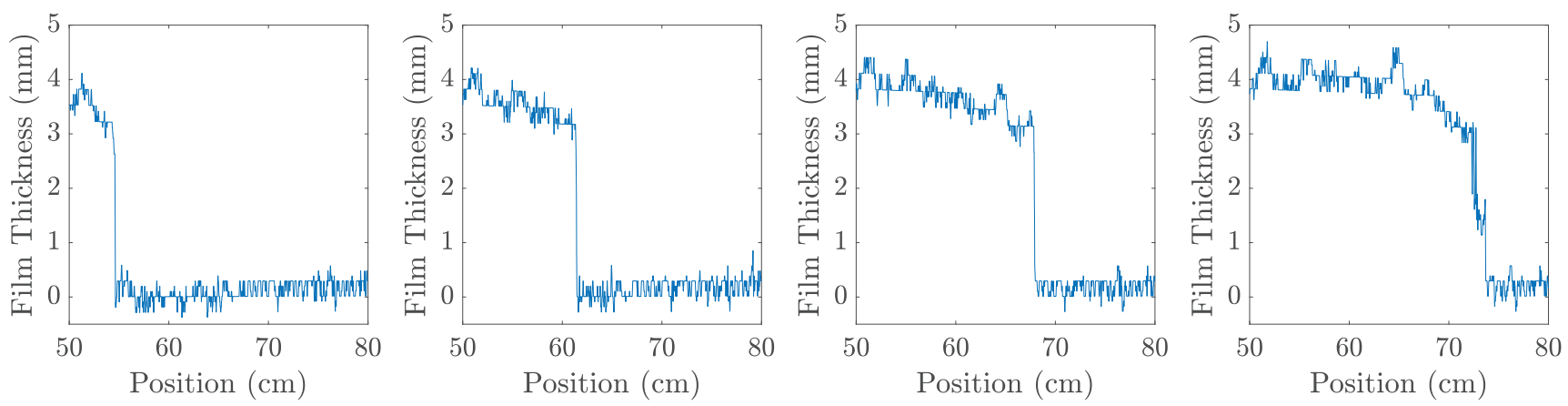

Figure 8. Film thickness of a constant flux slurry in the settled regime as it flows down an inclined track, with 30 seconds between consecutive graphs. These graphs correspond to the $(\chi, \alpha)=\left(0.880,20^{\circ}\right)$ experiment in Figure 6. The zero position is taken to be the location where the slurry starts to flow. Zero film thickness corresponds to positions on the track without slurry. 

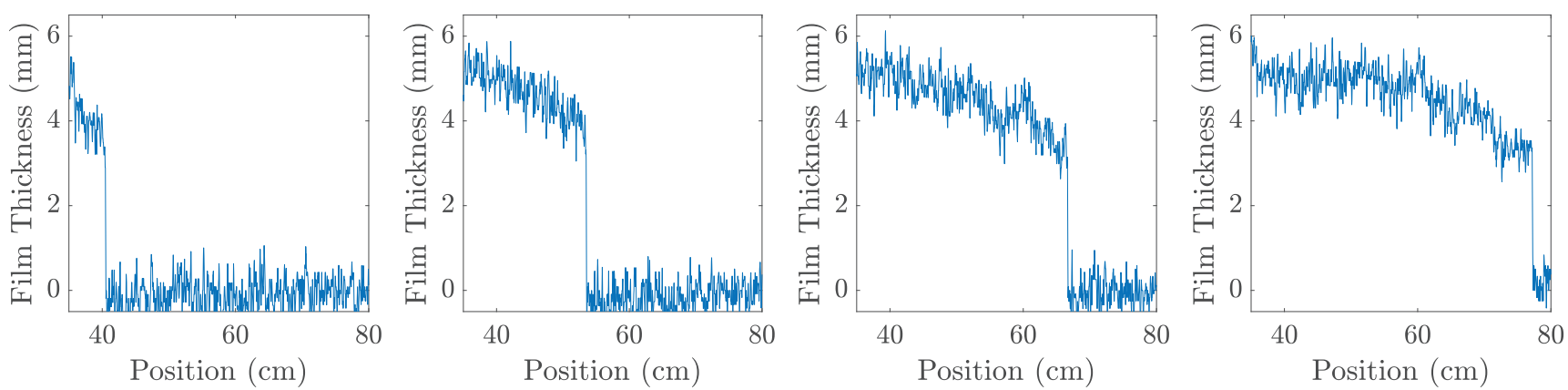

Figure 9. Film thickness of a constant flux slurry in the well-mixed regime as it flows down an inclined track, with 1 minute between consecutive graphs. These graphs correspond to the $(\chi, \alpha)=\left(0.378,20^{\circ}\right)$ experiment in Figure 6. The zero position is taken to be the location where the slurry starts to flow. Zero film thickness corresponds to positions on the track without slurry.
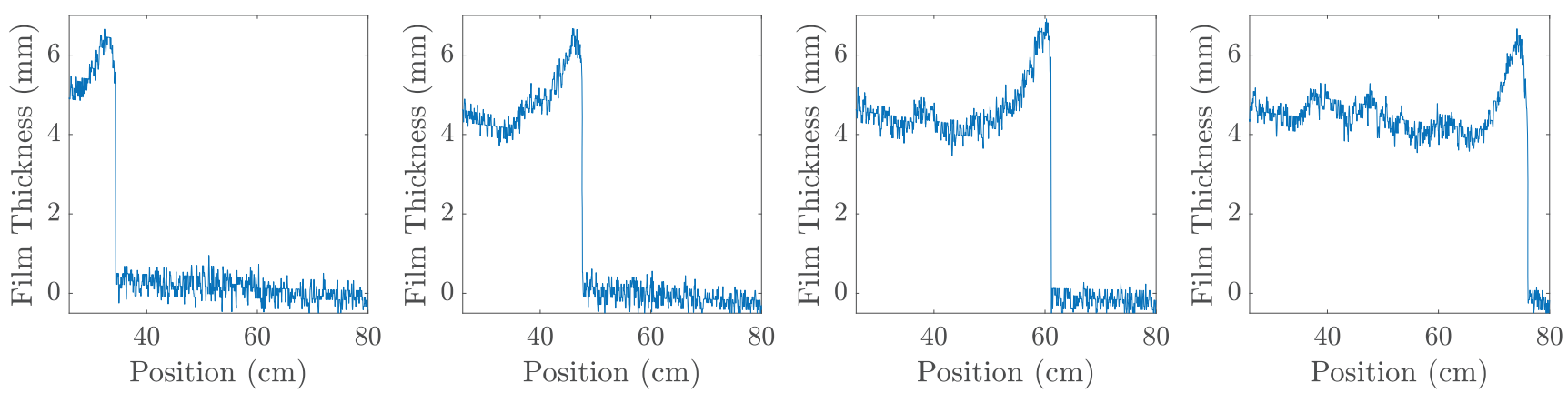

Figure 10. Film thickness of a constant flux slurry in the ridged regime as it flows down an inclined track, with 1 minute between consecutive graphs. These graphs correspond to the $(\chi, \alpha)=\left(0.889,50^{\circ}\right)$ experiment in Figure 6. The zero position is taken to be the location where the slurry starts to flow. Zero film thickness corresponds to positions on the track without slurry.

\section{CONCLUSIONS}

In this work we extended the existing literature on bidensity slurries flowing down an incline by analyzing both constant volume and constant flux initial conditions. We explicitly presented fitted fluid front positions and showed loose agreement with theoretical predictions ( $\sim t^{1 / 3}$ for constant volume and $\sim t^{1}$ for constant flux). Regime results were also presented for both initial conditions and showed that regardless of the initial condition, large particle volume fractions and inclination angles led to a ridged slurry while small corresponding values led to a settled slurry. The ratio of lighter particles to total particles, $\chi$, was shown to sometimes affect the regime of a constant volume slurry but had no effect on the regime of constant flux slurries, although a longer track would be required in future investigations to confirm this. Height profiles (film thicknesses) for constant flux initial conditions were similar for the settled and well-mixed regimes-a thin front increased into an approximate plateau-while the ridged regime exhibited distinct behavior-a tall leading ridge decreased into an approximate plateau.

\section{ACKNOWLEDGMENTS}

This work was funded by NSF grants DMS-1312543 and DMS-1659676. AB and CF were also funded by Simons Math $+\mathrm{X}$ Investigator Award number 510776. JB and AP were additionally funded by NSF grant CCF-1422795. MRL acknowledges the support of the Natural Sciences and Engineering Research Council of Canada (NSERC) [funding reference number PDF-502-479-2017]. Cette recherche a été financée par le Conseil de recherches en sciences naturelles et en génie du Canada (CRSNG) [numéro de référence PDF-502-479-2017]. 


\section{REFERENCES}

1. Huppert, H. E. (1982) Flow and instability of a viscous current down a slope, Nature 300, 427-429. https://doi.org/10.1038/300427a0

2. Cook, B. P., Bertozzi, A. L., and Hosio, A. E. (2008) Shock solutions for particle-laden thin films, SIAM J Appl Math 68(3), 760-783. https://doi.org/10.1137/060677811

3. Ward, T. , Wey, C. , Glidden, R., Hosoi, A. E., and Bertozzi, A. L. (2009) Experimental study of gravitation effects in the flow of a particle-laden thin film on an inclined plane, Phys Fluids 21(8), 083305. https://doi.org/10.1063/1.3208076

4. Murisic, N., Ho, J., Hu, V., Latterman, P., Koch, T., Lin, K., Mata, M., and Bertozzi, A. L. (2011) Particle-laden viscous thin-film flows on an incline: Experiments compared with a theory based on shear-induced migration and particle settling, Phys D 240(20), 1661-1673. https://doi.org/10.1016/j.physd.2011.05.021

5. Murisic, N., Pausader, B., Peschka, D., and Bertozzi, A. L. (2013) Dynamics of particle settling and resuspension in viscous liquid films, J Fluid Mech 717, 203-231. https://doi.org/10.1017/ffm.2012.567

6. Cook, B. P. (2008) Theory for particle settling and shear-induced migration in thin-film liquid flow, Phys Rev E 78(4), 045303. https://doi.org/10.1103/PhysRevE.78.045303

7. Wang, L., Mavromoustaki, A., Bertozzi, A. L., Urdaneta G., and Huang, K. (2015) Rarefaction-singular shock dynamics for conserved volume gravity driven particle-laden thin film, Phys Fluids 27(3), 033301. https://doi.org/10.1063/1.4913851

8. Zhou, J., Dupuy, A., Bertozzi, A. L., and Hosoi, A. E. (2005) Theory for shock dynamics in particle-laden thin films, Phys Rev Lett 94(11), 117803. https://doi.org/10.1103/PhysRevLett.94.117803

9. Lee, S., Stokes, Y., and Bertozzi, A. L. (2014) Behavior of a particle-laden flow in a spiral channel, Phys Fluids 26(4), 1661-1673. https://doi.org/10.1063/1.4872035

10. Arnold, D. J., Stokes, Y. M., and Green, J. E. F. (2015) Thin-film flow in helically-wound rectangular channels of arbitrary torsion and curvature, J Fluid Mech 764, 76-94. https://doi.org/10.1017/ffm.2014.703

11. Arnold, D. J., Stokes, Y. M., and Green, J. E. F. (2017) Thin-film flow in helically wound shallow channels of arbitrary cross-sectional shape, Phys Fluids 29(1), 013102. https://doi.org/10.1063/1.4973670

12. de Rooij, F. (2000) Sedimenting particle-laden flows in confined geometries, http://131.111.17.133/lab/people/sd103/papers/Theses/FransDeRooij1999.pdf (accessed Aug 2018).

13. Parsons, J. D., Whipple, K. X., and Simoni, A. (2001). Experimental study of the grain-flow, fluid-mud transition in debris flows, J Geol, 109(4), 427-447. https://doi.org/10.1086/320798

14. Wong, J. T., Lindstrom, M. and Bertozzi, A. L. (2019) Fast equilibration dynamics of viscous particle-laden flow in an inclined channel, J Fluid Mech 879, 28-53. https://doi.org/10.1017/jfm.2019.685

15. Richardson, J. F. and Zaki, W. N. (1954) The sedimentation of a suspension of uniform spheres under conditions of viscous flow, Chem Eng Sci 3(2), 65-73. https://doi.org/10.1016/0009-2509(54)85015-9

16. Schaflinger, U., Acrivos, A., and Zhang, K. (1990) Viscous resuspension of a sediment within a laminar and stratified flow, Int J Multiph Flow 16(4), 567-578. https://doi.org/10.1016/0301-9322(90)90017-D

17. Phillips, R. J., Armstrong, R. C., Brown, R. A., Graham, A. L., and Abbott, J. R. (1992) A constitutive equation for concentrated suspensions that accounts for shear-induced particle migration, Phys Fluids A 4(1), 30-40. https://doi.org/10.1063/1.858498

18. Leighton, D. and Acrivos, A. (1987) The shear-induced migration of particles in concentrated suspensions, J Fluid Mech 181, 415-439. https://doi.org/10.1017/S0022112087002155

19. Lee, S., Mavromoustaki, A., Urdaneta, G., Huang, K., and Bertozzi, A. L. (2014) Experimental investigation of bidensity slurries on an incline, Granul Matter 16(2), 269-274. https://doi.org/10.1007/s10035-013-0480-2

20. Lee, S., Wong, J., and Bertozzi, A. L. (2015) Equilibrium theory of bidensity particle-laden flows on an incline, in Mathematical Modelling and Numerical Simulation of Oil Pollution Problems (Ehrhardt, M., Ed.) 1st ed., 85-97, Springer, New York. https://doi.org/10.1007/978-3-319-16459-5 4 
21. Wong, J. T., and Bertozzi, A. L. (2016) A conservation law model for bidensity suspensions on an incline, Phys D 330(1), 47-57. https://doi.org/10.1016/j.physd.2016.05.002

\section{ABOUT THE STUDENT AUTHORS}

All undergraduate authors either currently attend or have graduated from the University of California, Los Angeles. Dominic Diaz graduated in June 2019 with a B.S. in Applied Mathematics and Physics and is currently pursuing a Ph.D. in Applied Mathematics at Cornell University. Jessica Bojorquez graduated in June 2018 with a B.S. in Mathematics in Computation and is currently a software engineer for Raytheon in Los Angeles, CA. Josh Crasto graduated in June 2018 with a B.S. in Applied Mathematics and is currently a software engineer for Macquarie Bank in Houston, TX. Margaret Koulikova graduated in June 2019 with a B.S. in Applied Mathematics and Statistics and is currently pursuing a Masters in Financial Mathematics at the University of Chicago. Tameez Latib will graduate in June 2020 with a B.S. in Applied Mathematics and Computer Engineering. Aviva Prins graduated in June 2019 with a B.S. in Applied Mathematics and is currently pursuing a Ph.D. in Computer Science at University of Maryland, College Park. Andrew Shapiro graduated in Winter 2019 with a B.S. in Applied Mathematics and is currently pursuing a Ph.D. in Statistics at UCLA. Clover Ye graduated in June 2018 with a B.S. in Applied Mathematics and Psychology and is currently pursuing a Masters in Mathematics at New York University.

\section{PRESS SUMMARY}

Particle-laden slurries persist in various settings, whenever particles are mixed or transported within a fluid. Here we investigate the properties of slurries with two particle species of different density mixed within a viscous fluid on an incline. We investigate two initial conditions-constant volume (a fixed amount of mixture) and constant flux (mixture being continuously pumped onto the incline)-and how they affect the phenomena that arise while flowing down the incline. Specifically, we fit for the position of the front of the fluid, fit for the thickness of the fluid (for the constant flux condition), and classify the qualitative features of the flow based on the location of the highest concentration of particles. These findings show that for both initial conditions the qualitative features of the flow can vary drastically depending on the number of particles within the mixture and the incline angle. 


\section{APPENDIX: FRONT POSITION AND FILM THICKNESS CODES}

We present a pseudocode for how we compute the front position of each individual fluid and particle front in Algorithm 1. In front tracking experiments, we placed the camera above the incline with the lens face parallel to the incline. Our track had a middle section for the slurry to flow down and a left section with equally-spaced measurement lines (see Figure 11a, a well-mixed slurry flowing down the incline). We present the cropped and rotated image of the slurry and measurement lines in Figure 11b. We present the same image with RGB values converted to HSV values and example clicks to find the maximum and minimum HSV values for each front in Figure 11c. For a settled slurry, the user should click 10-15 times for each distinct front.

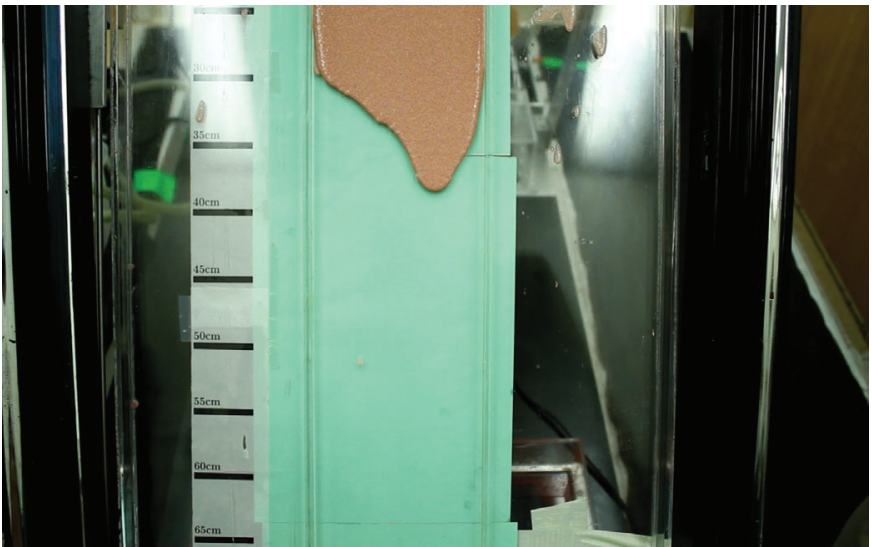

(a) unprocessed frame from video

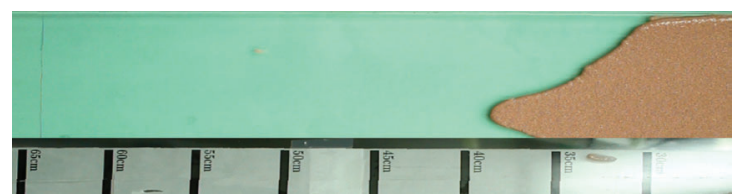

(b) rotated and cropped frame from video

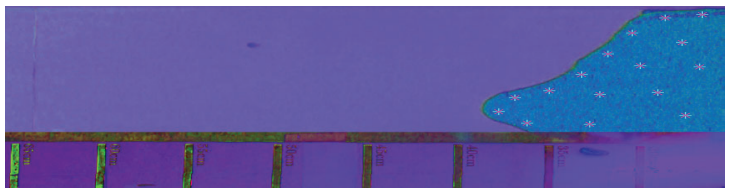

(c) HSV image with example clicking points

Figure 11. Images used in our front tracking code. (a) is an unprocessed image of the slurry flowing down the incline with measurement lines next to the slurry. (b) is a rotated and cropped version of (a). (c) is the same as (b) but with RGB values converted to HSV values and example clicks.

We present a pseudocode for how we compute the film thickness (height profile) of our slurries in Algorithm 2. To see example images that the code uses, see Figure 12. In these experiments, the camera must be in the same position for the refimage, cal_image, and experimental video-next to the incline, with the camera lens face perpendicular to the incline so that the camera can pick up the laser line deflection due to the calibration object and the slurry.

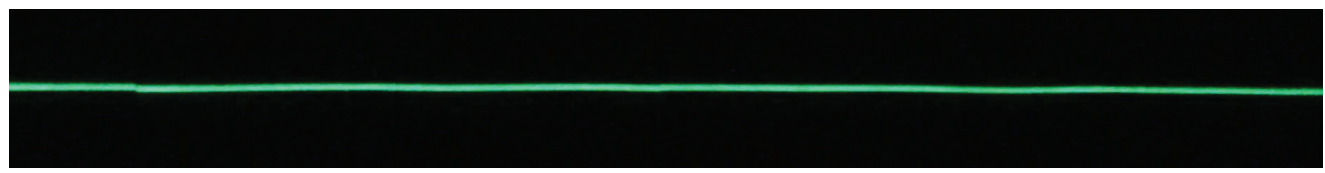

(a) example ref_image

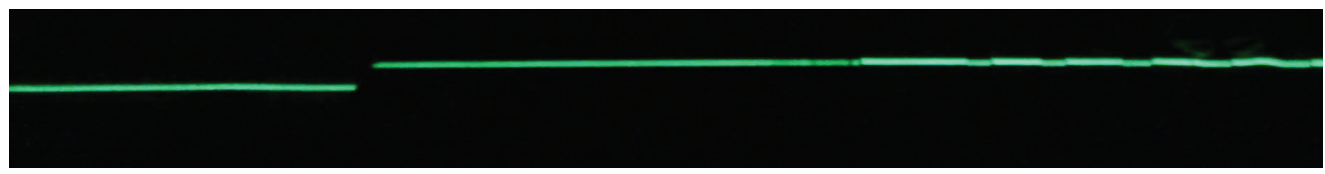

(b) example cal_image

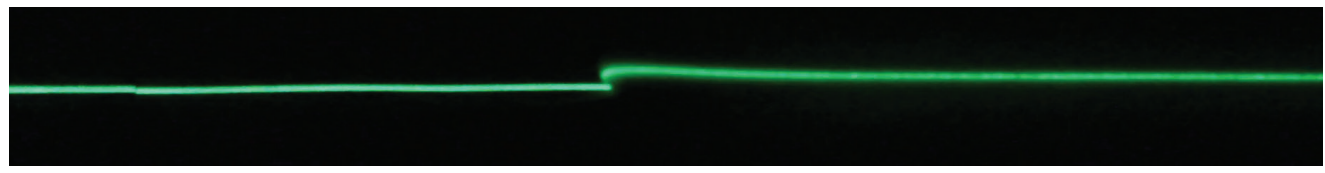

(c) example screenshot from experimental video

Figure 12. Images used in the height profile (film thickness) code. The three images are all taken from the same position and the slurry in (c) is flowing from right to left. 


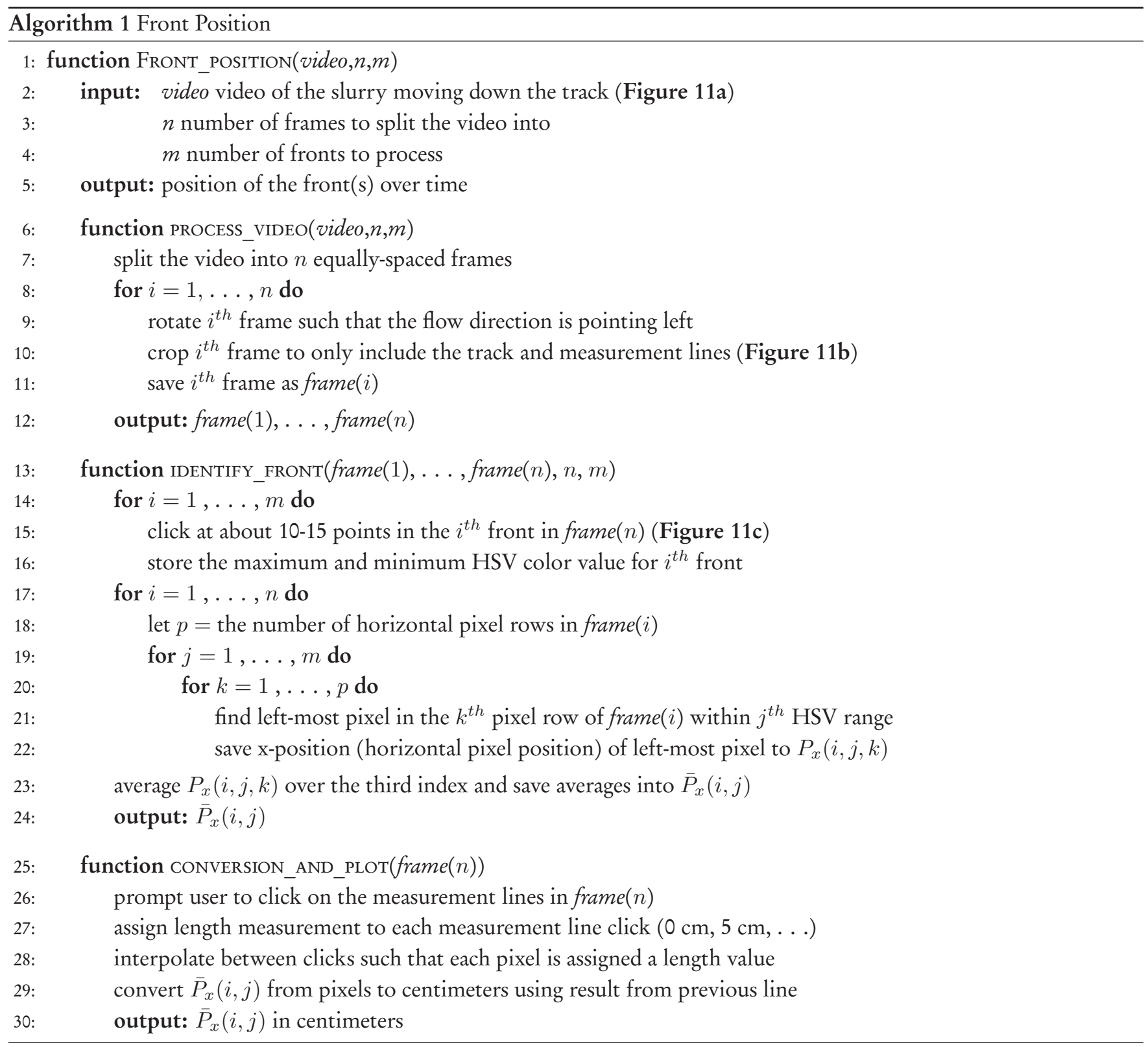




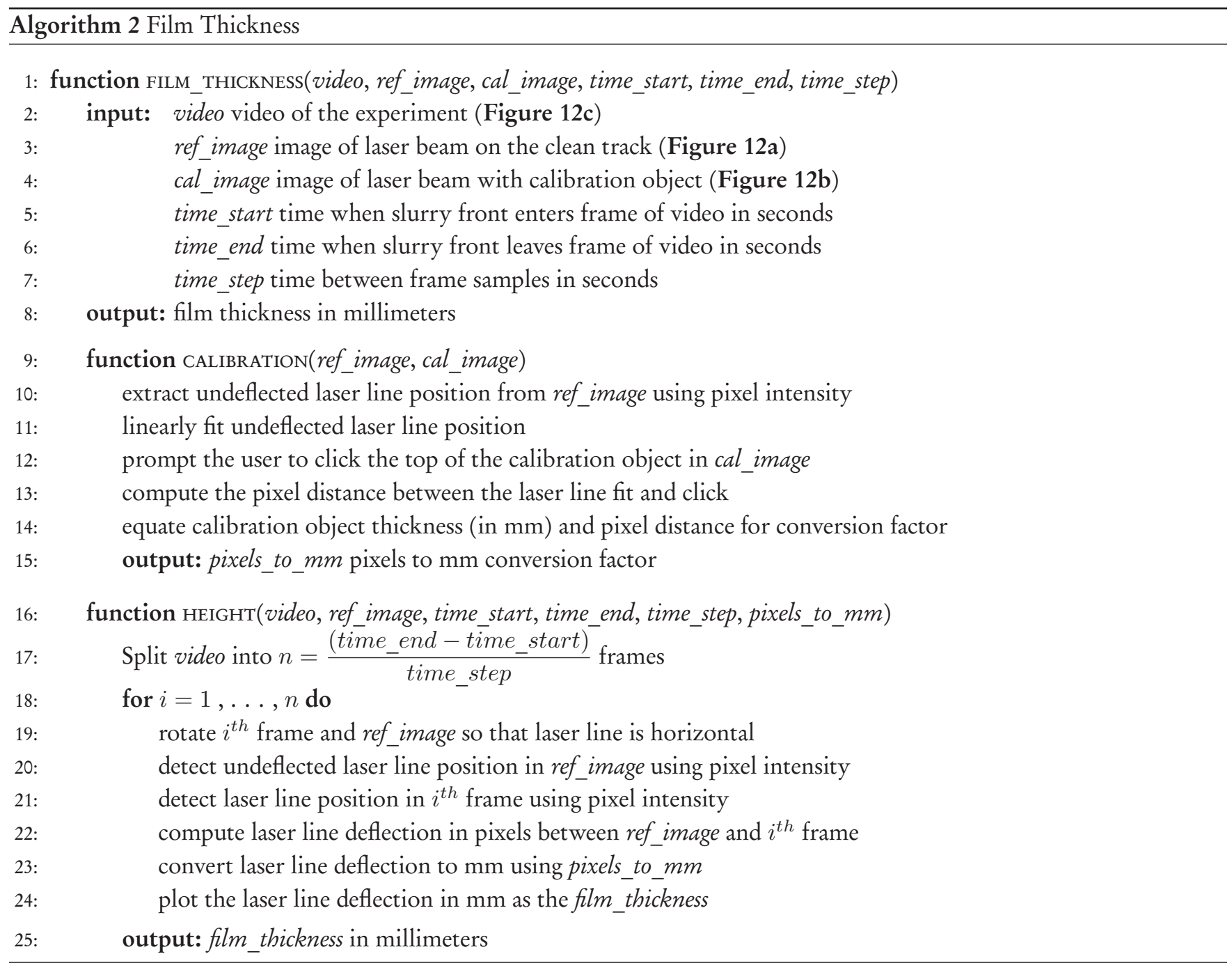

